\title{
Mutation Survey of the PHEX Gene and Oral Manifestation in a Chinese Family with X-linked Dominant Hypophosphatemic Ricket
}

Wang Yuanyuan, Chen Jie, Wu Nan, Zhao Yuming, Ge Lihong and Qin Man*

Department of Conservative Dentistry and Endodontics, School and Hospital of Stomatology, Peking University, Beijing, China

*Corresponding author: Qin Man, Department of Pediatric Dentistry, Peking University School and Hospital of Stomatology, \#22 Zhongguancun Nandajie, Haidian District, Beijing 100081, China, Tel: +8610 82195306; E-mail: qin-man@foxmail.com

Received date: October 28, 2016; Accepted date: December 08, 2016; Published date: December 16, 2016

Copyright: (C) 2016 Yuanyuan W, et al. This is an open-access article distributed under the terms of the Creative Commons Attribution License, which permits unrestricted use, distribution, and reproduction in any medium, provided the original author and source are credited.

\begin{abstract}
Objective: X-linked dominant hypophosphatemic rickets is the most common genetic disorder of renal phosphate wasting with an approximate prevalence of 1 in 20,000 in humans. Until now, no more than 15 mutations in the PHEX gene have been reported in Chinese patients with familial XLH among the all the 338 PHEX mutations. The aim of this study is describe the oral manifestation of a Chinese XLH family, analyze the dentin structure alteration and gene mutation.
\end{abstract}

Methods: In this XLH family, the oral manifestation was described by panoramic tomograph and oral photographs, dentin structure alteration was studied by both tooth section and 3D morphology measurement laser microscope, the gene mutation was studied by PCR technique.

Results: Pulp infection and teeth loss were facilitated in XLH patients due to the anatomy change, hypophosphatemic dentin alterations in deciduous teeth displayed as large interglobular spaces between the unmerged calcospherites. A nonsense gene mutation c.1645 (p.Arg549X) was detected in this study.

Conclusions: We found a PHEX gene mutation in this XLH Chinese family. Our findings are useful for understanding the oral manifestation, dentin alteration and genetic basis of Chinese patients with XLH.

Keywords: X-linked dominant hypophosphatemic rickets; 3D morphology measurement laser microscope; Dentin structure; Gene mutation; Genetic diseases

\section{Introduction}

$\mathrm{X}$-linked dominant hypophosphatemic rickets (XLHOMIM\#307800), with an approximate prevalence of 1 in 20,000 in humans, is the most common genetic disorder of renal phosphate wasting. The main characteristics of hypophosphatemic rickets are defective renal phosphate re-absorption and abnormal bone mineralization. The clinical features of this disease include short stature, low extremity deformities, bone and/or joint pain, calcification of ligaments, and dental abnormalities. Three main types of hypophosphatemic rickets have been reported, X-linked dominant hypophosphatemia (XLH), autosomal dominant hypophosphatemic rickets (ADHR), and autosomal recessive hypophosphatemic rickets (ARHR) which are associated with mutations in the phosphateregulating endopeptidase gene (PHEX), the fibroblast growth factor 23 gene (FGF23), and the dentin matrix acidic phosphoprotein 1 gene (DMP1), respectively.

Since the PHEX gene has been reported, currently 338 mutations have been found in XLH patients (http://www.phexdb.mcgill.ca; Thu August 4 02:45:45 2016), which mostly occur in European (Northern European), North American, and Far Eastern populations [1]. However, no more than 15 mutations in the PHEX gene have been reported in Chinese familial with XLH [2-6]. PHEX mutations include deletions, insertions, and base-pair substitutions have been previously described, all of which are predicted to be loss of function mutations. Although the genetic cause of XLH has been established, the mechanism has yet to be elucidated, some research have reported that PHEX may inactivate bone mineralization inhibitors and that one of the extra-osseous consequences of PHEX inactivation leads to an increase in the level of FGF-23 [7,8]. The dentin alteration of XLH has been reported before [8], however no system description of oral manifestation and gene mutations in XLH families has been reported before. In this study, we aimed to study the oral manifestation, dentin structure by a $3 \mathrm{D}$ morphology measurement laser microscope and gene mutation in a XLH family.

\section{Materials and Methods}

\section{Patients}

This study was approved by the Ethics Committee of the School and Hospital of Stomatology, Peking University. The subjects provided informed consent before participating in the study, and clinical data and DNA samples were obtained.

The proband is a 5-year-old boy, presenting oral caries and tooth pain. The boy was born of a non-consanguineous marriage, and had a birth weight of $3.4 \mathrm{~kg}$. At the time of presentation to us, he presented with short stature $(90 \mathrm{~cm})$, mild lower extremity genuvalgum deformities. The proband's mother, a 30 years old woman, also presented to us, her height was $135 \mathrm{~cm}$, her lower extremity deformities were much more severe and her dental condition was poor with many teeth lost. The boy and his mother were diagnosed with 
Citation: Yuanyuan W, Jie C, Nan W, Yuming Z, Lihong G, et al. (2016) Mutation Survey of the PHEX Gene and Oral Manifestation in a Chinese Family with X-linked Dominant Hypophosphatemic Ricket. Dentistry 6: 402. doi:10.4172/2161-1122.1000402

Page 2 of 6

XLH based on their medical histories, clinical manifestations, physical examination, laboratory indices and radiologic evidence. The proband's father is healthy. The general features are shown in Table 1.
The pedigrees of the families with X-linked hypophosphatemic rickets are shown in Figure 1.

\begin{tabular}{|c|c|c|c|c|c|c|c|c|}
\hline Family & Gender & Age (yr) & Height $(\mathrm{cm})$ & Weight (Kg) & BMI & Clinical findings & Gene mutation & Inheritance \\
\hline \multirow{2}{*}{ I-son } & \multirow{2}{*}{ M } & \multirow{2}{*}{5} & \multirow{2}{*}{90} & \multirow{2}{*}{20} & \multirow{2}{*}{24.7} & Genu varum & EXON15 & \multirow{2}{*}{ familial } \\
\hline & & & & & & odontodysplasia & c. $1645 \mathrm{CT}$ & \\
\hline \multirow{4}{*}{ I-mother } & \multirow{4}{*}{$\mathrm{F}$} & \multirow{4}{*}{30} & \multirow{4}{*}{135} & \multirow{4}{*}{40} & \multirow{4}{*}{21.9} & Genu varum & EXON15 & \multirow{4}{*}{ familial } \\
\hline & & & & & & odontodysplasia & c. $1645 \mathrm{CT}$ & \\
\hline & & & & & & bone pain & & \\
\hline & & & & & & teeth falling out & & \\
\hline
\end{tabular}

Table 1: General features for the XLH patients studied.

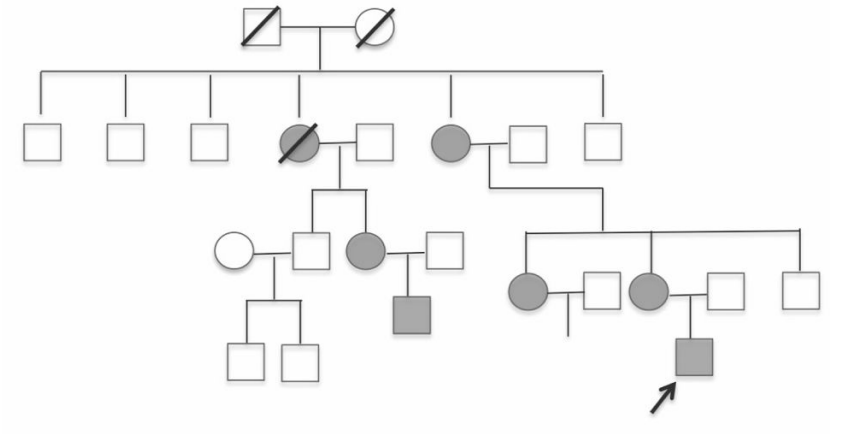

Figure 1: Family Pedigrees. Black symbol shows an affected individual for hypophosphatemic rickets, and the open symbols represent the unaffected individuals. The circles and squares indicate females and males, respectively. The arrow shows the proband in this family.

\section{Tooth collection}

Retained deciduous teeth were collected from the proband and healthy children (aged 5-7 years). All teeth were obtained with the parents' and children's informed consent. All the teeth were individually mounted with self-cure acrylic resin. It was then mounted on a milling machine for preparing teeth section. Sectioned teeth were prepared for a light microscope (Nikon ECLIPSE TS-100) analysis and 3D morphology measurement laser microscope (Keyence VK-X200, Japan).

\section{Mutation analysis}

Genomic DNA was isolated from peripheral blood leukocytes using the conventional phenol-chloroform extraction method. We screened the PHEX gene completely for mutations in this family (including the proband and his parents), The DNA sequence for the PHEX gene was obtained from available online database (NCBI Reference) and PCR and sequencing primers were designed by Primer Premier 3.0. All 22 exons and exon-intron boundaries in the PHEX gene were amplified by polymerase chain reaction (PCR).

Hot Start PCR reaction was performed in our study, and Takara DNA polymerase (Takara Bio Inc.) was used for highly specific amplification in hot-start PCR reaction. The cycling program of amplification was 96 or 5 minutes, 10 cycles of 96 for 20s, 58 for 2 minutes, 72 for 30s. Thirty-five cycles of 96 for 20s, 48 for 30s, 72 for 30 s.

Direct sequencing was performed using the BigDye Terminator Cycle Sequencing Ready Reaction Kit, version 3.1 (Applied Biosystems, Foster, CA, USA), and the cycling program of sequencing was 95 for 15s, 35 cycles of 95 for $15 \mathrm{~s}, 50$ for 5s, 60 for 90 s, 72 for 1 minute, 72 for 2 minutes. The resulting PCR products were directly sequenced using an automated ABI PRISM 3130 sequencer (Foster, CA) (Table 2).

\begin{tabular}{|l|l|l|l|}
\hline Primer & Primer sequence (F) 5'-3' & Primer sequence (R) 5'-3' & Product size \\
\hline PHEX-exon1 & GGAAAGCCAAGGCAACC & GAACGCAGGCAAACAGC & 448 \\
\hline PHEX-exon2 & TCTTGGAGTGGCTTTC & GGAGCCTTGCTTAGAG & 798 \\
\hline PHEX-exon3 & CAAGGCTTGGAAACTGGTTGA & CTTATGTTGAGATCTGGGAGTCC & 457 \\
\hline PHEX-exon4 & GGCTCCGTTGAGAATA & TGCCCTGCTGACTTTG & 750 \\
\hline PHEX-exon5 & CACATTGAAGCGTGGAT & AGTGAGGAGGCAAGGAC & 784 \\
\hline PHEX-exon6 & TACCTTGGGAAAGTCAC & TAGATAAGGGCATTGAG & 658 \\
\hline PHEX-exon7 & GCACCATACTTTGAG & TTACTTCCTAGAGGG & 459 \\
\hline
\end{tabular}


Citation: Yuanyuan W, Jie C, Nan W, Yuming Z, Lihong G, et al. (2016) Mutation Survey of the PHEX Gene and Oral Manifestation in a Chinese Family with X-linked Dominant Hypophosphatemic Ricket. Dentistry 6: 402. doi:10.4172/2161-1122.1000402

Page 3 of 6

\begin{tabular}{|c|c|c|c|}
\hline PHEX-exon8 & TGCCTAAAGTGATGT & CACAGACAAGGGATA & 402 \\
\hline PHEX-exon9 & TCTGGATGGCAATGATCAGGA & ATCACAAAGGACACCGGGAT & 459 \\
\hline PHEX-exon10 & CAGACACGAAAGGCTGTG & ATGGGAATACACTAATAAGACG & 698 \\
\hline PHEX-exon11 & TACCTTTGCCTCTACTATCT & TACCTGCTAACTCATCCTC & 462 \\
\hline PHEX-exon12 & GCTGGTCTCAAACTCC & CCATGTGCCTCTTACA & 664 \\
\hline PHEX-exon13 & TGCCCTTCACAGTGGCTTGC & ACCCACTCAGCTACGCATCGTT & 440 \\
\hline PHEX-exon14 & ATGGAACGCATTGTAAA & СTCTGACTTGAGGGGAATTACA & 285 \\
\hline PHEX-exon15 & GGACAGCCCTTTAGATT & СTTCCTTCACCAGCATA & 506 \\
\hline PHEX-exon16 & GCCATGTATGAAGGAGC & TAGCCAAACTACACTCTGC & 727 \\
\hline PHEX-exon17 & TTTATGGGTATGGTTATTCG & AAGCCATCACAGCAAGA & 377 \\
\hline PHEX-exon18 & GTGAACACTTTGGGTC & AAACACGCTTTGAGAA & 545 \\
\hline PHEX-exon19 & TCCAGAGCACCTTGCTGAGGAT & AGGGTCAATGGGGAGACACACTT & 413 \\
\hline PHEX-exon20 & СCTGCTAGGAATGGAAA & TGGTGGCATAAAGTCAG & 782 \\
\hline PHEX-exon21 & TCCTCAGTATAATTTGGAGCAGT & GGAAATCACACGTCCACAATG & 440 \\
\hline PHEX-exon22 & AAAACTGGCAAGTCCTA & GGTCACCTACCCAAATA & 589 \\
\hline
\end{tabular}

Table 2: Primer information for PCR amplication of PHEX gene.

\section{Results}

\section{Clinical features of subjects}

The general features of the patients are shown in Table 1 and Figure $2 \mathrm{~A}$. The radiology results of the patients are shown in Figure $2 \mathrm{~B}$ and $2 \mathrm{C}$, genu varum with an "O" appearance were shown and the proband's mother had more severe phenotypes beside a short stature.
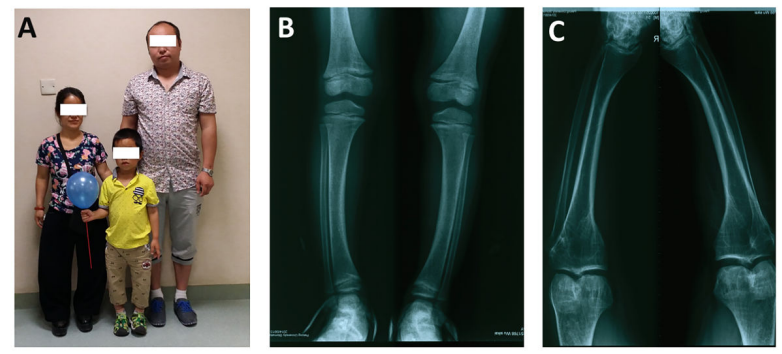

Figure 2: The outward appearance and radiological evidence of $\mathrm{XLH}$ patients. (A) the picture shows genu varum with an "O" appearance and short stature of the proband and his mother. (B-C) the radiograph shows his lower extremity genu varus deformities, the mother $(\mathrm{C})$ has a more severe deformity than the proband $(\mathrm{B})$.

\section{Oral manifestation of subjects}

The panoramic tomograph of the proband and his family were shown in Figure 3, the proband's mother had a very poor oral condition with many teeth lost and serious periodontitis of the residual teeth (Figure 3A). The osseous structures of both the proband and his mother showed less dense trabeculations. The oral condition of the proband was shown in Figure 4. The clinical examination revealed an anterior crossbite, a primary dentition with some tooth decays, gingival fistula and unusual abscess. His oral hygiene was poor. Radiographic examination revealed large pulp chambers with an abnormally high pulp/tooth volume ratio.
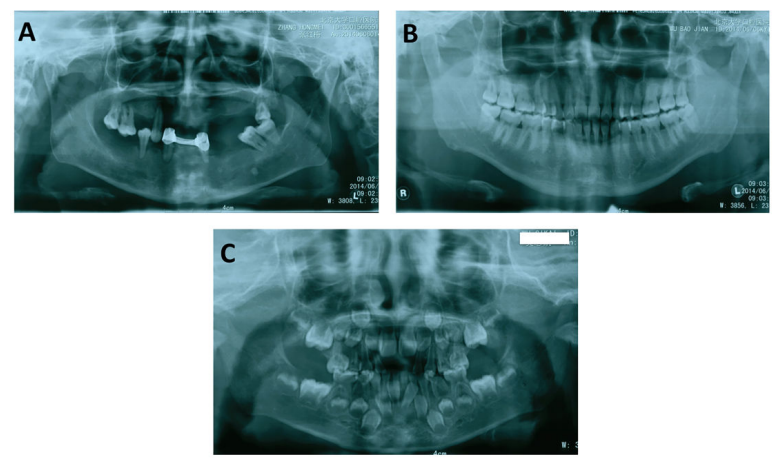

Figure 3: The panoramic tomograph of the XLH family. (A-C) The proband's mother has many teeth lost and serious periodontitis of the residual teeth (A). The panoramic tomograph of the proband's father shows no obvious abnormal (B). The proband has a mixed dentition (C). The osseous structures of both the proband and his mother show less dense trabeculations. 
Citation: Yuanyuan W, Jie C, Nan W, Yuming Z, Lihong G, et al. (2016) Mutation Survey of the PHEX Gene and Oral Manifestation in a Chinese Family with X-linked Dominant Hypophosphatemic Ricket. Dentistry 6: 402. doi:10.4172/2161-1122.1000402

Page 4 of 6

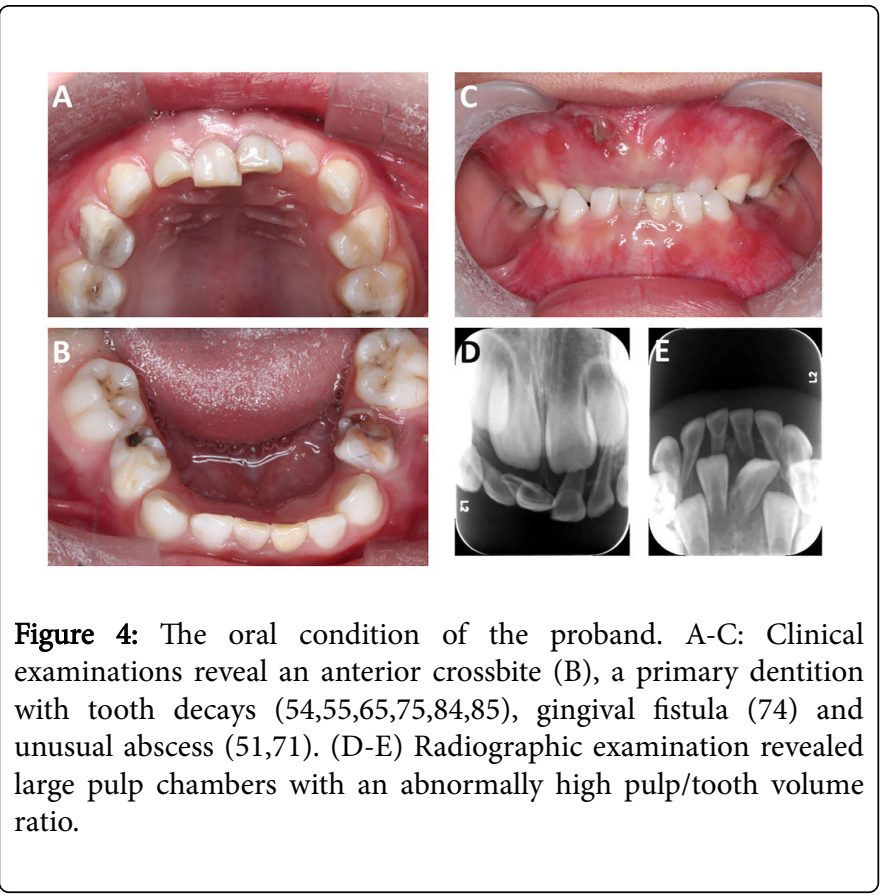

\section{Dentin structure alteration}

Under the light microscope, the structure of enamel and mantle dentin is similar in controls and the proband. The circumpulpal dentin from the hypophosphatemic patient showed an altered structure (Figure 5).
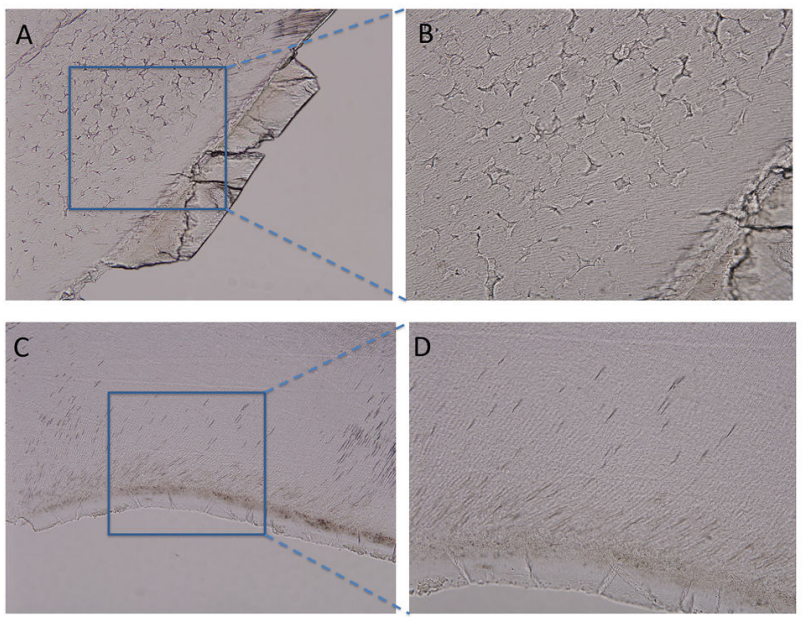

Figure 5: Dentin structure alteration by light microscope. The structure of enamel and mantle dentin is similar in proband $(A, B)$ and the controls $(C, D)$. Magnifying figures shows the circumpulpal dentin from the proband shows an altered structure (B) compared to the controls (D).

Under the 3D morphology measurement laser microscope, hypophosphatemic dentin displayed severe alterations in deciduous teeth. Large interglobular spaces were observed between unmerged calcospherites (Figure 6A and 6C), while continuous dentin tubules regularly crossed a homogeneous dentin in control teeth (Figure 6B and $6 \mathrm{D})$.

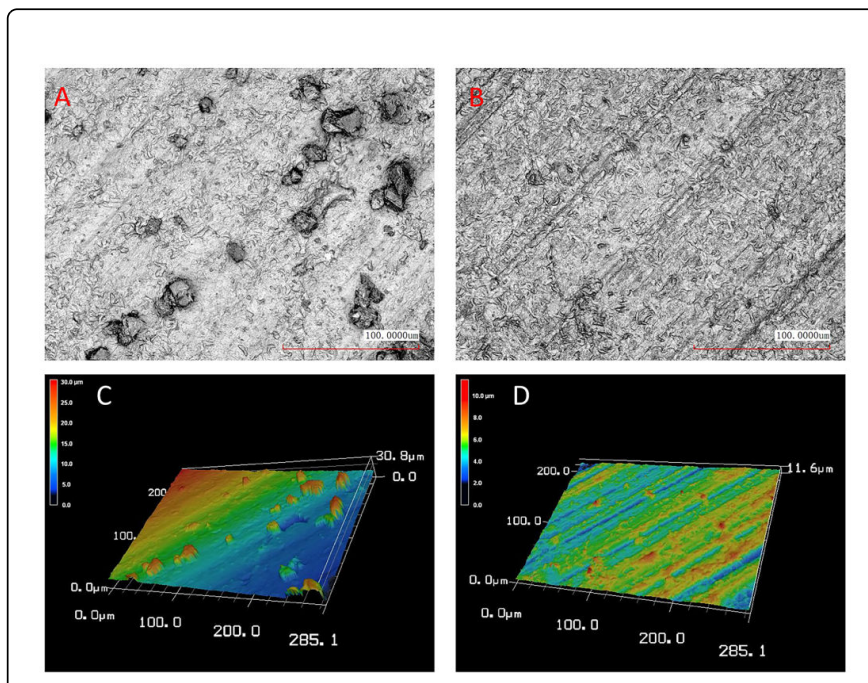

Figure 6: Dentin structure alteration by 3D morphology measurement laser microscope. Large interglobular spaces are observed between unmerged calcospherites (Figure 6A and 6C), while continuous dentin tubules regularly cross a homogeneous dentin in control teeth (Figure 6B and 6D).

\section{Gene mutation}

In this XLH family, a nonsense mutation of exon 15 was presented in the proband and his mother.

This mutation was a $\mathrm{C}$ to $\mathrm{T}$ transition at c.1645, which changed codon CGA to TGA and transformed an arginine codon to a stop codon (Figure 7).

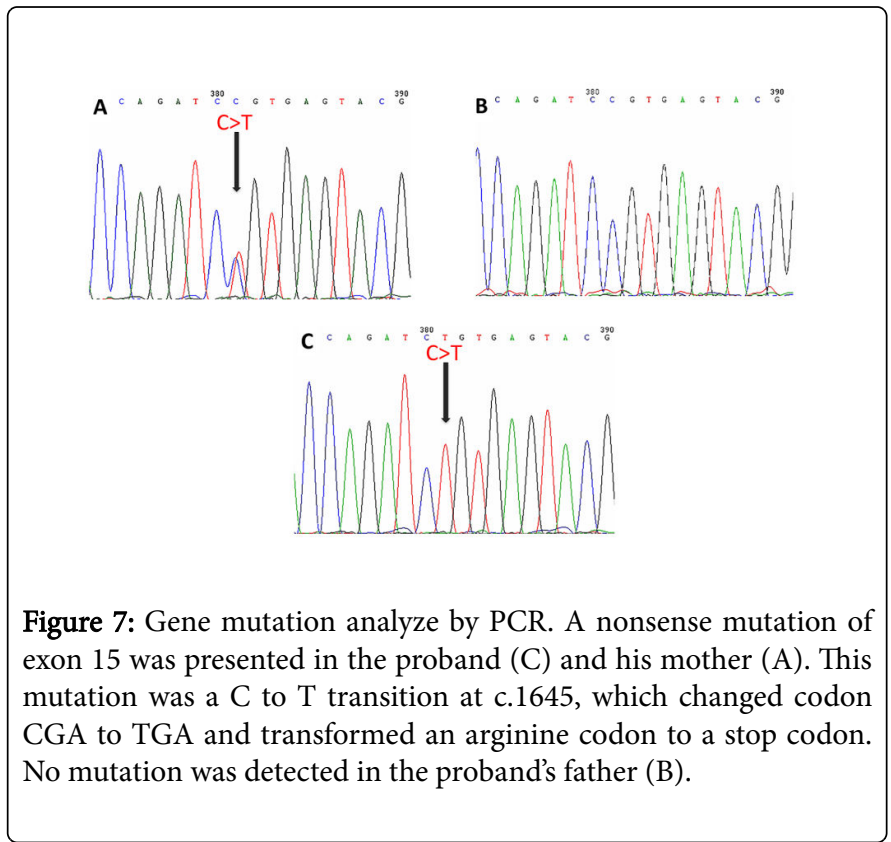




\section{Discussion}

In this study, we described the oral manifestation of a Chinese XLH family, studied the dentin structure of the deciduous teeth and identified a PHEX mutation.

The clinical signs of XLH can vary from one patient to another owing to several factors: family history, the degree of hypophosphatemia and the age of the patient at the start of systemic treatment. The main symptoms are severe bone deformities, particularly a characteristic bowing of the legs. Dental anomalies and delayed eruptions are systematically found among these patients and affect both primary and permanent dentitions [9-13]. In our case, no delayed eruption was found. On radiographs, the osseous structures show less dense trabeculations, particularly on the alveolar bone, and the lamina dura is often absent [14].

We used a 3D morphology measurement laser microscope to observe the morphology of XLH dentin structure, which is more intuitive compared to the scanning electron microscope. The dentin structure we observed was similar as it has been described before $[10,15,16]$ : the circumpulpal dentin exhibits major differences and the presence of large interglobular spaces are resulting from the lacking fusion of calcospherites. Human dentin mineralization is a consecutive process that occurs by growth and fusion of calcospherites on the mineralization front. The interglobular spaces are filled with organic matrix that has not been mineralized. Only the mantle dentin is not affected by this disease, as its mineralization is independent of noncollagenous protein phosphorylation [15]. It has been reported that abnormal expression and distribution of noncollagenous proteins in hypophosphatemic dentin and liberation of aspartate serine-rich motif (ASARM) peptides may partially explain the impaired dentin mineralization associated with the disease [17]. Progression of bacteria to the pulp could be facilitated by the anatomy and prominence of the large pulp horns, the presence of enamel and dentin cracks, and the wear of thinner enamel. One of the prominent clinical signs is the spontaneous emergence of multiple acute or chronic dental abscesses, with no history of dental trauma or decays [18-20]. This situation always occurs in the child with primary teeth, and often follows the natural sequence of tooth eruption [21].

A PHEX mutation was detected in this XLH family, this nonsense mutation was an $\mathrm{C}$ to $\mathrm{T}$ transition at c.1645 (p.Arg549X), which changed codon CGA to TGA and transformed an arginine codon to a stop codon. This mutation has been reported before [22,23], but is first reported in the Chinese population. It is reported a same mutation in different races can cause quite different clinical features. For example, p.Trp444X was firstly reported by Beck-Nielsen SS [24] in a sporadic patient, a Danish male, with a normal height, mild skeletal and endodontic phenotype. Whereas, in the study of Yue et al. [25], the mutation was found in familial patients with abnormal gait, kyphosis, and hip and knee joint pain. In our study, the clinical features were similar to previous report in p.Arg549X, with leg deformities, tooth abnormalities, height deficiency. Based on these researches, relationship between the phenotype and genotype of the PHEX gene is variant and the relationship between the phenotype and genotype of the PHEX gene is also complex. Yuan et al. [26] had provided evidences that aberrant PHEX function in osteoblasts and/or osteocytes alone was sufficient to result in the HYP-mouse phenotype. The conclusion of Davidai's research [27] was that the phenotypic heterogeneity common to XLH might be due to genetic variability partly, and they suspected that such heterogeneity was due to altered phosphorus transport at variable segments of the proximal convoluted tubule. The HYP Consortium [28] had also proposed that the PHEX gene was involved in XLH. Popowska et al. [22] mentioned that phosphate and vitamin D3 supplementation could slow down the progressive growth retardation and leg bowing.

\section{Conclusion}

In conclusion, we report a Chinese XLH family in this study, the dentin structure and gene mutation were studied. 3D dentin structure was first describe in this study, more studies will be needed in future for analyzing the phenotype-genotype relationship in Chinese population and compared it to the cases in other races.

\section{References}

1. Sabbagh Y, Jones AO, Tenenhouse HS (2000) PHEXdb, a locus-specific database for mutations causing X-linked hypophosphatemia. Hum Mutat 16: 1-6.

2. Yang L, Yang J, Huang X (2013) PHEX gene mutation in a Chinese family with six cases of X-linked hypophosphatemic rickets. J Pediatr Endocrinol Metab 26: 1179-1183.

3. Xia W, Meng X, Jiang Y, Li M, Xing X, et al. (2007) Three novel mutations of the PHEX gene in three Chinese families with X-linked dominant hypophosphatemic rickets. Calcif Tissue Int 81: 415-420.

4. Lo FS, Kuo MT, Wang CJ, Chang CH, Lee ZL, et al. (2006) Two novel PHEX mutations in Taiwanese patients with X-linked hypophosphatemic rickets. Nephron Physiol 103: p157-p163.

5. Kang QL, Xu J, Zhang Z, He JW, Lu LS, et al. (2012) Three novel PHEX gene mutations in four Chinese families with $\mathrm{X}$-linked dominant hypophosphatemic rickets. Biochem Biophys Res Commun 423: 793-798.

6. Qiu G, Liu C, Zhou J, Liu P, Wang J, et al. (2010) Prenatal diagnosis for a novel splice mutation of PHEX gene in a large Han Chinese family affected with X-linked hypophosphatemic rickets. Genet Test Mol Biomarkers 14: 385-391.

7. Addison WN, Nakano Y, Loisel T, Crine P, McKee MD (2008) MEPEASARM peptides control extracellular matrix mineralization by binding to hydroxyapatite: an inhibition regulated by PHEX cleavage of ASARM. J Bone Miner Res 23: 1638-1649.

8. Boukpessi T, Septier D, Bagga S, Garabedian M, Goldberg M, et al. (2006) Dentin alteration of deciduous teeth in human hypophosphatemic rickets. Calcif Tissue Int 79: 294-300.

9. Baroncelli GI, Angiolini M, Ninni E, Galli V, Saggese R, et al. (2006) Prevalence and pathogenesis of dental and periodontal lesions in children with X-linked hypophosphatemic rickets. Eur J Paediatr Dent 7: 61-66.

10. Murayama T, Iwatsubo R, Akiyama S, Amano A, Morisaki I (2000) Familial hypophosphatemic vitamin D-resistant rickets: dental findings and histologic study of teeth. Oral Surg Oral Med Oral Pathol Oral Radiol Endod 90: 310-316.

11. Pereira CM, de Andrade CR, Vargas PA, Coletta RD, de Almeida OP, et al. (2004) Dental alterations associated with X-linked hypophosphatemic rickets. J Endod 30: 241-245.

12. Seow WK, Needleman HL, Holm IA (1995) Effect of familial hypophosphatemic rickets on dental development: a controlled, longitudinal study. Pediatr Dent 17: 346-350.

13. Abe K, Ooshima T, Lily TS, Yasufuku Y, Sobue S (1988) Structural deformities of deciduous teeth in patients with hypophosphatemic vitamin D-resistant rickets. Oral Surg Oral Med Oral Pathol 65: 191-198.

14. Resnick D (1998) Implant placement and guided tissue regeneration in a patient with congenital vitamin D-resistant rickets. J Oral Implantol 24: 214-218.

15. Chaussain-Miller C, Sinding C, Septier D, Wolikow M, Goldberg M, et al. (2007) Dentin structure in familial hypophosphatemic rickets: benefits of vitamin D and phosphate treatment. Oral Dis 13: 482-489. 
Citation: Yuanyuan W, Jie C, Nan W, Yuming Z, Lihong G, et al. (2016) Mutation Survey of the PHEX Gene and Oral Manifestation in a Chinese Family with X-linked Dominant Hypophosphatemic Ricket. Dentistry 6: 402. doi:10.4172/2161-1122.1000402

Page 6 of 6

16. Hillmann G, Geurtsen W (1996) Pathohistology of undecalcified primary teeth in vitamin D-resistant rickets: review and report of two cases. Oral Surg Oral Med Oral Pathol Oral Radiol Endod 82: 218-224.

17. Gaucher C, Boukpessi T, Septier D, Jehan F, Rowe PS, et al. (2009) Dentin noncollagenous matrix proteins in familial hypophosphatemic rickets. Cells Tissues Organs 189: 219-223.

18. Larmas M, Hietala EL, Simila S, Pajari U (1991) Oral manifestations of familial hypophosphatemic rickets after phosphate supplement therapy: a review of the literature and report of case. ASDC J Dent Child 58 328-334.

19. Seow WK (2003) Diagnosis and management of unusual dental abscesses in children. Aust Dent J 48: 156-168.

20. Goodman JR, Gelbier MJ, Bennett JH, Winter GB (1998) Dental problems associated with hypophosphataemic vitamin D resistant rickets. Int J Paediatr Dent 8: 19-28.

21. McWhorter AG, Seale NS (1991) Prevalence of dental abscess in a population of children with vitamin D-resistant rickets. Pediatr Dent 13 91-96.

22. Popowska E, Pronicka E, Sulek A, Jurkiewicz D, Rowinska E, et al. (2001) $\mathrm{X}$-linked hypophosphatemia in Polish patients. 2. Analysis of clinical features and genotype-phenotype correlation. J Appl Genet 42: 73-88.
23. Rowe PS, Oudet CL, Francis F, Sinding C, Pannetier S, et al. (1997) Distribution of mutations in the PEX gene in families with $\mathrm{X}$-linked hypophosphataemic rickets (HYP). Hum Mol Genet 6: 539-549.

24. Beck-Nielsen SS, Brixen K, Gram J, Brusgaard K (2012) Mutational analysis of PHEX, FGF23, DMP1, SLC34A3 and CLCN5 in patients with hypophosphatemic rickets. J Hum Genet 57: 453-458.

25. Yue H, Yu JB, He JW, Zhang Z, Fu WZ, et al. (2014) Identification of two novel mutations in the PHEX gene in Chinese patients with hypophosphatemic rickets/osteomalacia. PLoS One 9: e97830.

26. Yuan B, Takaiwa M, Clemens TL, Feng JQ, Kumar R, et al. (2008) Aberrant Phex function in osteoblasts and osteocytes alone underlies murine X-linked hypophosphatemia. J Clin Invest 118: 722-734.

27. Davidai GA, Nesbitt T, Drezner MK (1990) Normal regulation of calcitriol production in Gy mice. Evidence for biochemical heterogeneity in the X-linked hypophosphatemic diseases. J Clin Invest 85: 334-339.

28. Francis F, Hennig S, Korn B, Reinhardt R, de Jong P, et al. (1995) A gene (PEX) with homologies to endopeptidases is mutated in patients with Xlinked hypophosphatemic rickets. The HYP Consortium. Nat Genet 11: 130-136. 